BMJ Open

Diabetes

Research

\& Care

\section{Estimating the effects of second-line therapy for type 2 diabetes mellitus: retrospective cohort study}

Assaf Gottlieb, Chen Yanover, Amos Cahan, Yaara Goldschmidt
To cite: Gottlieb A, Yanover C, Cahan A, et al. Estimating the effects of second-line therapy for type 2 diabetes mellitus: retrospective cohort study. BMJ Open Diab Res Care 2017;5:e000435. doi:10.1136/ bmjdrc-2017-000435

- Additional material is published online only. To view please visit the journal online (http://dx.doi.org/10.1136/ bmjdrc-2017-000435).

Received 25 April 2017 Revised 3 October 2017 Accepted 11 October 2017

\section{CrossMark}

Machine Learning for Healthcare and Life Sciences, IBM Research, Haifa, Israel

Correspondence to Prof Assaf Gottlieb; assaf.gottlieb@uth.tmc.edu and Dr Chen Yanover; cheny@il.ibm.com

\section{ABSTRACT}

Objective Metformin is the recommended initial drug treatment in type 2 diabetes mellitus, but there is no clearly preferred choice for an additional drug when indicated. We compare the counterfactual drug effectiveness in lowering glycated hemoglobin ( $\mathrm{HbA} 1 \mathrm{C})$ levels and effect on body mass index (BMI) of four diabetes second-line drug classes using electronic health records. Study design and setting Retrospective analysis of electronic health records of US-based patients in the Explorys database using causal inference methodology to adjust for patient censoring and confounders.

Participants and exposures Our cohort consisted of more than 40000 patients with type 2 diabetes, prescribed metformin along with a drug out of four second-line drug classes-sulfonylureas, thiazolidinediones, dipeptidyl peptidase 4 (DPP-4) inhibitors and glucagon-like peptide-1 agonists—during the years 2000-2015. Roughly, 17000 of these patients were followed for 12 months after being prescribed a second-line drug.

Main outcome measures $\mathrm{HbA} 1 \mathrm{C}$ and $\mathrm{BMI}$ of these patients after 6 and 12 months following treatment. Results We demonstrate that all four drug classes reduce $\mathrm{HbA1c}$ levels, but the effect of sulfonylureas after 6 and 12 months of treatment is less pronounced compared with other classes. We also estimate that DPP-4 inhibitors decrease body weight significantly more than sulfonylureas and thiazolidinediones.

Conclusion Our results are in line with current knowledge on second-line drug effectiveness and effect on BMI. They demonstrate that causal inference from electronic health records is an effective way for conducting multitreatment causal inference studies.

\section{INTRODUCTION}

Type 2 diabetes mellitus (T2DM) affects more than 29 million people in the USA and is the seventh leading cause of death. ${ }^{12}$ The American Diabetes Association Standards of Medical Care, ${ }^{3}$ supported by several studies, ${ }^{45}$ recommends dietary changes and physical exercise as the initial treatment, followed by administration of metformin if lifestyle changes fail to reach glycemic control. According to the Standards of Medical Care, if metformin does not achieve glycemic target within 3 months, one of the following six second-line medications should be added: sulfonylureas (SU),

\section{Significance of this study}

What is already known about this subject?

- The effects of type 2 diabetes second-line drugs on glycosylated hemoglobin levels and on body mass index (BMI) have been evaluated in clinical studies. However, the clinical implication of these studies is limited by small number of participating individuals and the homogeneity of the study populations.

- Meta-analysis studies have increased sample size but potentially suffer from similar homogeneity biases.

\section{What are the new findings?}

- This study performs, for the first time, a large-scale analysis of the therapeutic and adverse effects of type 2 diabetes second-line drugs in real-world population using electronic health records.

- We confirm current knowledge for glycosylated hemoglobin levels, while showing better effects on decreasing BMI for inhibitors of dipeptidyl peptidase 4 (DPP-4).

How might these results change the focus of research or clinical practice?

- Our results show that while sulfonylureas are the most commonly prescribed second-line drugs, their estimated reduction in glycosylated hemoglobin levels is significantly smaller than the estimated effects of thiazolidinediones, glucagon-like peptide-1 agonists or DPP-4 inhibitors. DPP-4 inhibitors also show significant reduction of BMI compared with sulfonylureas and thiazolidinediones.

- We demonstrated that causal inference methods can confirm and expand current knowledge in a cost-effective way and should gain increased focus when addressing epidemiological questions.

thiazolidinediones (TZD), inhibitors of dipeptidyl peptidase 4 (DPP-4), glucagon-like peptide-1 receptor agonists (GLP-1), sodium-glucose cotransporter 2 (SGLT2) inhibitors or insulin. Currently, the guidelines do not prefer one class over the others. The effectiveness, costs and risk of complication of those drug classes were compared in clinical trials ${ }^{6}$ and meta-analyses of their results. ${ }^{7-9}$ 
These comparisons found no significant difference in drug class effect on the percentage of blood glycated hemoglobin (HbAlc); thus, no specific recommendation about the choice of a second drug could be made. ${ }^{10}$

Notably, clinical trials are laborious and costly. Trials often include small samples with limited representativeness of the target population (eg, between 2005 and 2012, the Food and Drug Administration approved drugs based on a median number of 2 clinical trials and the median number of patients enrolled was $760^{11}$ ). Meta-analyses of clinical trials may have higher power and be more generalizable, but are also vulnerable to publication bias, smallstudy effects and limited degree of heterogeneity. ${ }^{12}$

Electronic health records (EHRs) hold promise as an alternative approach to conduct causal inference experiments that can address some of these imitations. ${ }^{13} 14$ Specifically, secondary use of EHRs requires lower costs, can scale to a large number of patients and better represents the heterogeneity in the population. There are trade-offs to using the EHR approach, and such analyses may suffer from three major limitations: First, patients may get treatment outside the institutions included in the EHR, resulting in missing and fragmented data. ${ }^{15}$ Second, confounders play a crucial role in effect size estimation and their identification is challenging. ${ }^{16}$ Third, differences in protocols or adoption rate for new medications across institutions may obscure true effect size and might not be generalizable beyond the database from which they are derived. ${ }^{17}$

Here, our aim is to compare the effects of T2DM second-line drugs using a real-world evidence approach. We emulate a multiarm clinical trial of four classes of drugs for diabetes, commonly used as second-line treatment (SU, TZD, DPP-4 and GLP-1). We compare the counterfactual (ie, potential) effectiveness (in terms of HbA1c levels) and body mass index (BMI) outcomes of 17082 patients over the course of 12 months, adjusting for confounders and censoring (additional 23789 patients). For reference, a recent meta-analysis of antidiabetes drugs ${ }^{8}$ was based on data of about 18000 patients. We describe the measures we have taken to address the aforementioned limitations of causal inference from EHR data. Our results are in line with current knowledge, thus demonstrating that causal inference from EHRs is an effective way for conducting multitreatment causal inference studies.

\section{RESEARCH DESIGN AND METHODS}

\section{Study design}

\section{Data source}

We used the Explorys database (IBM), which includes EHR records of approximately 50 million patients, pooled from multiple different healthcare systems in the USA. Data consist of a combination of clinical EHRs, healthcare system outgoing bills and adjudicated payor claims, are standardized and normalized using common ontologies, including SNOMED and The National Drug File
- Reference Terminology, and are searchable through a Health Insurance Portability and Accountability Act of 1996-enabled, de-identified database tools. The EHR data include patient demographics, diagnoses, procedures, prescribed drugs, vitals and laboratory values.

\section{Cohort definition}

We defined a cohort of patients with T2DM based on the Northwestern University diabetes phenotyping algorithm, ${ }^{18}$ comprising 40871 patients, using the following criteria:

\section{Inclusion criteria}

Our analysis considered T2DM patients, identified by having at least two types of evidence for T2DM, out of T2DM diagnosis, T2DM-specific drugs, and indicative lab values (fasting and random glucose or HbA1c levels). We included only patients who were first prescribed metformin and subsequently prescribed, during the years 2000-2015, a second-line drug belonging to any of four classes: SU, TZD, inhibitors of DPP-4 and GLP-1 agonists (online supplementary table 1 lists drugs for each drug class).

The first prescription with order status marked as completed (ie, that it was not canceled or erroneous) of the second-line drug was considered the 'index-date' (emulating the date of intervention allocation and initiation in clinical trials).

We required the patients to have at least 12 months of documented pretreatment observation period prior to the index date.

\section{Exclusion criteria}

Patients with type 1 diabetes mellitus, identified by either a type 1 diabetes diagnosis code or prescription of pramlintide (approved also for patients with T2DM who use insulin), as well as patients prescribed more than one second-line drug classes on the index date were excluded from the analysis. Our analysis did not include the following three second-line medications: SGLT2 inhibitors, meglitinides and $\alpha$-glucosidase inhibitors due to the low number of patients receiving it within our data $(185$, 429 and 153 patients with available HbA1c measurements, respectively). We did not compare insulin because it is not commonly considered as the first choice for secondline therapy in clinical practice; it is also administered to patients with more advanced or severe disease than oral agents ${ }^{1}$; and patient acceptance of insulin often involves unique psychological and social factors that are not part of our cohort data, nor usually recorded in the EHR. These factors are likely to be important confounders and no analysis that uses this type of data could adjust for them.

\section{Outcomes and follow-up time}

As outcomes, we used HbAlc and BMI at two follow-up periods, 6 and 12 months after the index date. We averaged the HbAlc and BMI over \pm 3 month windows for each period (3-9 months for the first follow-up period 


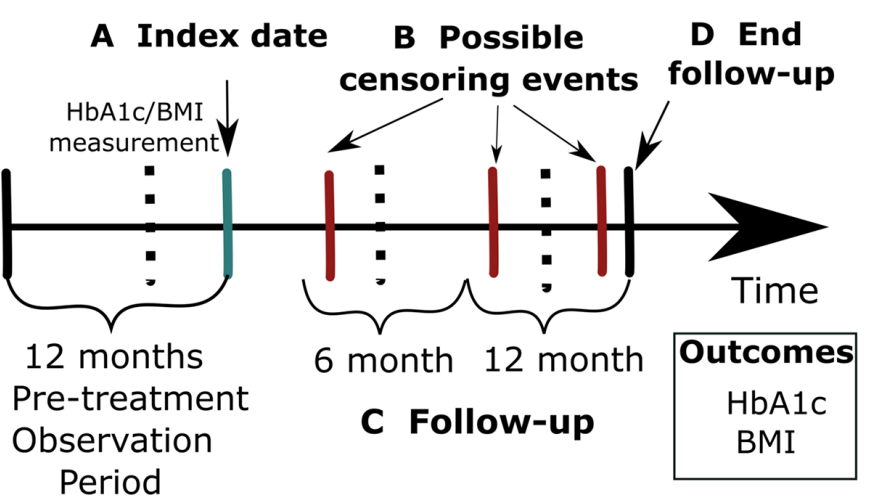

Figure 1 Illustration of the causal inference scheme. (A) Index date is the first prescription of diabetes second-line drug after use of metformin. (B) Potential censoring events include switching to another second-line drug, missing glycated hemoglobin (HbA1c) or body mass index (BMI) measurement, or undergoing bariatric surgery. (C) Outcomes $(\mathrm{HbA} 1 \mathrm{c}$ and $\mathrm{BMI})$ are checked after 6 and 12 months from index date. (D) Follow-up ends after 15 months.

and 9-15 months for the second; figure 1). We chose a 12-month pretreatment observation period to ensure that the second-line drug is prescribed for the first time as, for example, $99 \%$ of patients receiving SU have prescription period $<12$ months. It also balances the need for a complete and stable baseline (ie, longer period) with the need to include more patients and avoid bias due to exclusion of patients with relatively limited histories in our data (ie, a shorter period). We chose follow-up periods of 6 and 12 months (averaging over \pm 3 month windows, resulting in 15 months in total) since they provide a good estimate of the short to intermediate effects of the drugs and correspond to the majority of random clinical trial follow-up time interval. ${ }^{68}$ We required each patient to have at least one HbAlc measurement during the pretreatment observation period.

\section{Analysis methods}

We considered two potential biases: (1) selection bias due to censoring; and (2) confounders, affecting both treatment choice and measured outcome (HbAlc levels or BMI).

In order to handle these two biases, we extracted patient characteristics within the pretreatment observation window using the feature engineering framework of Ozery-Flato et al. ${ }^{19}$ The comprehensive set of features included demographic information (age, sex, ethnicity), insurance type, patient-aggregated diagnoses using Clinical Classifications Software categories, categories of Charlson ${ }^{20}$ and Elixhauser comorbidity indexes, ${ }^{21}$ prescribed drugs (active ingredients), and laboratory results values over the baseline period. Diagnosis codes and drugs are binary features (measuring the existence of a diagnosis or a drug prescription for that patient). Categorical features, such as insurance type or ethnicity, were split into binary features. For lab values, we included the number of times a lab value was measured within the baseline period, and the maximal, minimal and average values within that period. For the HbA1C outcome, we also included the last measurement before treatment and the time from first diabetes diagnosis (based on the Northwestern University diabetes phenotyping algorithm). As a preliminary step, we filtered features that were dominated ( $>95 \%$ of patients) by a single value or were spurious ( $>80 \%$ with missing values), resulting in 632 features. Missing lab values were imputed using the median value of the test across patients.

\section{Censoring analysis}

For both HbAlc and BMI inference, we considered patients as censored if they received a second-line treatment, but during the follow-up period (1) had no 6-month or 12-month HbA1C or BMI measurements; (2) switched or added another antidiabetic drug (including the following drug classes, which were not directly evaluated in this work: insulin, SGLT2 inhibitors, meglitinides and $\alpha$-glucosidase inhibitors); or (3) underwent bariatric surgery. We corrected for censoring by reweighing the uncensored population using inverse probability of censoring weighting (IPCW) ${ }^{22}$

\section{Confounder analysis}

We defined the set of confounders in two ways: (1) domain expert confounder set, manually selected by an internist, aided by literature search; and (2) a comprehensive confounder set, treating all the 632 extracted features as confounders. In total, we selected 34 domain expert confounders for HbA1c inference (online supplementary

\begin{tabular}{|c|c|c|c|c|c|}
\hline Drug class & Patients (n) & Treatment change $^{*}$ & Missing outcome* & Average age $\dagger$ & $\%$ Female $^{\star}$ \\
\hline Sulfonylurea & 26684 & $4336\left(16 \%, 3 e^{-152}\right)$ & $12269(46 \%,-)$ & $61.2\left(2 \mathrm{e}^{-98}\right)$ & $47.7 \%\left(2 \mathrm{e}^{-15}\right)$ \\
\hline Thiazolidinedione & 4794 & $1145\left(24 \%, 2 \mathrm{e}^{-12}\right)$ & $2235(47 \%,-)$ & $59.6(0.001)$ & $48.2 \%(-)$ \\
\hline $\begin{array}{l}\text { Glucagon-like peptide-1 } \\
\text { receptor agonists }\end{array}$ & 1532 & $398\left(26 \%, 4 e^{-9}\right)$ & $735(48 \%,-)$ & $52.8\left(5 \mathrm{e}^{-113}\right)$ & $66.6 \%\left(3 e^{-44}\right)$ \\
\hline Dipeptidyl peptidase 4 & 7861 & $2314\left(29 \%, 3 e^{-118}\right)$ & $3405\left(43 \%, 5 e^{-6}\right)$ & $58.9\left(2 e^{-32}\right)$ & $51.1 \%\left(8 e^{-5}\right)$ \\
\hline
\end{tabular}

Per-confounder statistics appear in online supplementary table 2.

${ }^{*}$ Proportion test. Missing entries $(-)$ are not significant with FDR $<0.05$.

†Wilcoxon rank-sum test. Missing entries $(-)$ are not significant with FDR $<0.05$.

FDR, false discovery rate; HbA1c, glycated hemoglobin; T2DM, type 2 diabetes mellitus. 
Table 2 Descriptive statistics of patients on T2DM second-line drug classes for the BMI outcome

\begin{tabular}{|c|c|c|c|c|c|}
\hline Drug class & Patients (n) & Treatment change* $^{*}$ & Missing outcome* & Average age $†$ & $\%$ Female $^{*}$ \\
\hline Sulfonylurea & 18170 & $2967\left(16 \%, 2 \mathrm{e}^{-109}\right)$ & $8611\left(47 \%, 2 e^{-16}\right)$ & $60.9\left(4 e^{-17}\right)$ & $48.4 \%(0.01)$ \\
\hline Thiazolidinedione & 2691 & $640\left(23.8 \%, 3 e^{-6}\right)$ & $1503\left(56 \%, 2 e^{-29}\right)$ & $59.2\left(7 e^{-05}\right)$ & $49.3 \%(-)$ \\
\hline $\begin{array}{l}\text { Glucagon-like peptide-1 } \\
\text { receptor agonists }\end{array}$ & 1172 & $293\left(25 \%, 5 e^{-5}\right)$ & $441\left(38 \%, 3 e^{-8}\right)$ & $52.9\left(3 e^{-86}\right)$ & $66.6 \%\left(7 e^{-34}\right)$ \\
\hline Dipeptidyl peptidase 4 & 6295 & $1852\left(29 \%, 3 e^{-92}\right)$ & $2352\left(37 \%, 2 e^{-49}\right)$ & $58.7\left(2 e^{-29}\right)$ & $50.9 \%(0.003)$ \\
\hline
\end{tabular}

Per-confounder statistics appear in online supplementary table 3.

${ }^{*}$ Proportion test. Missing entries $(-)$ are not significant with FDR $<0.05$.

†Wilcoxon rank-sum test. Missing entries $(-)$ are not significant with FDR $<0.05$.

BMI, body mass index; FDR, false discovery rate; T2DM, type 2 diabetes mellitus.

table 2) and 8 domain expert confounders for BMI inference (online supplementary table 3). We used doubly robust (DR) estimator suggested by Robins et $a l^{23}$ to correct for confounders. This estimator combines a model for the distribution of the counterfactual outcome (standardization) and a treatment assignment mechanism model (inverse probability of treatment weighting, IPTW). As demonstrated by Bang and Robins, ${ }^{24}$ DR estimators improve on either estimators because they are consistent even when only one of the models is correctly specified. This makes DR especially suited for observational data, where one can never be sure that either model is correct. For the outcome model, we used ridge regression with fivefold cross-validation to adjust the regularization coefficient. For the treatment model, we used multiclass logistic regression with the regularization strength set to one and using balancing of the class sizes. Next, similar to Gerhard $e t a l^{25}$ we multiplied the IPCW weights obtained from the censoring model with the IPTW weights from the treatment model, and capped weights smaller than the first and larger than the 99th percentiles to their corresponding percentiles, as suggested by Cole and Hernán. ${ }^{26}$ Capping the weights trims the tails of the distribution of the inverse probability weighted estimator, reduces instability and was shown to work better than removing the concerned units altogether. ${ }^{27}$ We then fed these weights into the outcome model to compute the DR estimator.

Based on Groenwold $e t a l,{ }^{28}$ we also tested the results of stratification of continuous variables, for example, age and lab values, into five categories to prevent introduction of residual confounding. We obtained similar counterfactual mean values to the non-categorized values but larger confidence intervals (CIs) and thus omitted these results for brevity.

As suggested by Austin,$^{29}$ the standardized difference, $d$, can be used to quantify covariate imbalance across subject groups. Specifically, for continuous confounders:

$$
d=\frac{\bar{\chi}_{\text {treatment }}-\bar{\chi}_{\text {control }}}{\sqrt{\frac{S_{\text {treatment }}^{2}+S_{\text {control }}^{2}}{2}}}
$$

where $\bar{\chi}_{\text {treatment }}$ and $\bar{\chi}_{\text {control }}$ denote the sample mean of the covariate in treated and untreated subjects, respectively, whereas $S_{\text {treatment }}^{2}$ and $S_{\text {control }}^{2}$ denote the sample variance of the covariate in treated and untreated subjects, respectively.

And, for dichotomous confounders:

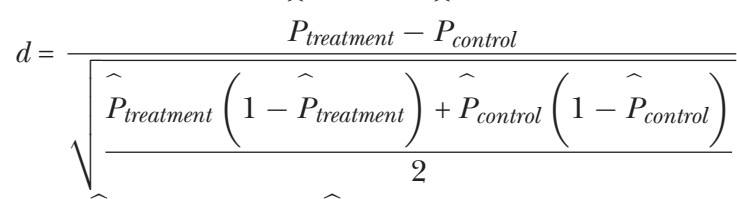

where $\mathrm{P}_{\text {treatment }}$ and $\mathrm{P}_{\text {control }}$ denote the prevalence or mean of the dichotomous variable in treated and untreated subjects, respectively.

We followed Austin ${ }^{29}$ and tested for imbalance in the confounders after correcting for the treatment models and censoring by comparing the number of confounders that were below the 0.1 threshold before and after weighing. For additional validation, we applied our inference scheme to two negative controls ${ }^{30}$ : patient height and pretreatment $\mathrm{HbA1C}$, which are unaffected by treatment type. For the outcome of pretreatment $\mathrm{HbA1C}$, we excluded pretreatment HbA1C from the set of confounders.

\section{Patient involvement}

No patients were involved in setting the research question or the outcome measures, nor were they involved in developing plans for design or implementation of the study. No patients were asked to advise on interpretation or writing up of results. There are no plans to disseminate the results of the research to study participants or the relevant patient community.

\section{RESULTS}

\section{Study design}

Our cohort included 40871 patients. Of these, 28328 also had available BMI before the prescription of secondline drugs and were used for inference of counterfactual BMI (tables 1 and 2, online supplementary figure 2-3). There were significantly more censored patients on TZD, GLP-1 or DPP-4 who switched or added another drug than patients on $\mathrm{SU}$ (censored patients, $\mathrm{p}<2 \mathrm{e}^{-109}$; tables 1 and 2). TZD and SU had significantly higher percentage of patients with missing BMI measurements during the follow-up than GLP-1 and DPP-4 $\left(\mathrm{p}<3 \mathrm{e}^{-8}\right.$; table 2). 

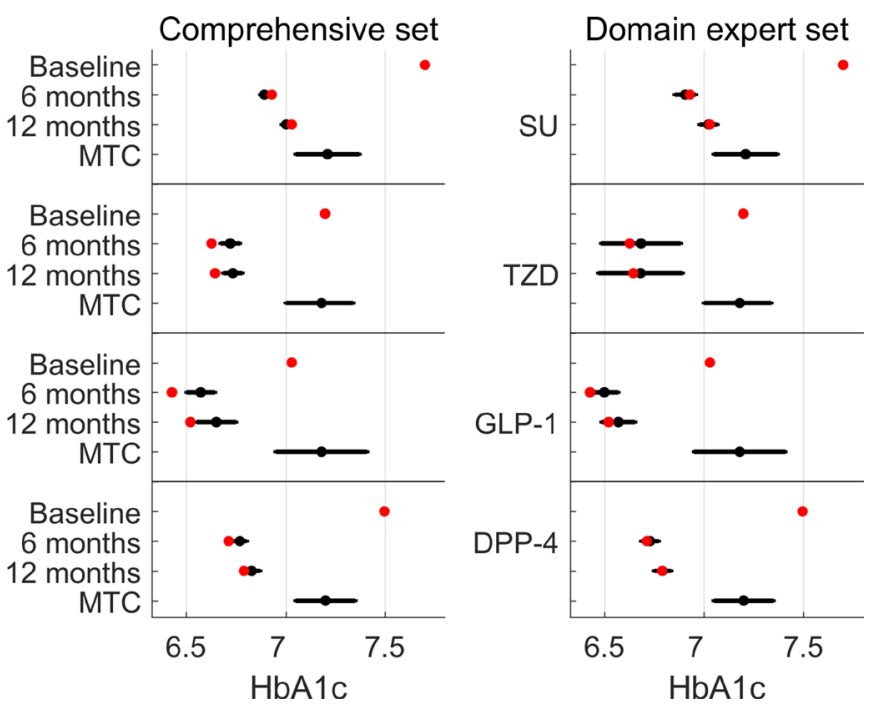

Figure 2 Predicted and observed $\mathrm{HbA} 1 \mathrm{c}$ levels using doubly robust estimation adjusting for either a comprehensive set of confounders (left panel) or a set of confounders provided by a domain expert (right panel). Red dots indicate the actual measurements of patients at baseline (before second-line treatment), after 6 and 12 months. Black dots (with error bars) represent the counterfactual predictions and $95 \% \mathrm{Cls}$, supposing all patients were treated with that drug class. The results of the Bayesian mixed-treatment comparison (MTC) meta-analysis by Mclntosh et al ${ }^{78}$ are marked MTC. DPP-4, dipeptidyl peptidase 4; GLP-1, glucagon-like peptide-1 receptor agonists; HbA1c, glycated hemoglobin; SU, sulfonylurea; TZD, thiazolidinedione.

Finally, the patients on GLP-1 were about 6 years younger on average $(p \sim 0)$ and included significantly higher rate of women $\left(\mathrm{p}<3 \mathrm{e}^{-44} ;\right.$ table 1$)$. The patient age distribution (online supplementary figure 1) is similar to the age distribution published by the Centers for Disease Control and Prevention (CDC) for 2011. ${ }^{31}$

\section{Analysis methods}

We applied causal inference methods to compute the counterfactual HbAlc levels and BMI (for each one of the four drug classes) at each of the two follow-up time points, adjusting for censored patients and confounders (Research design and methods).

Our balancing test (methods) showed that the percentage of balanced confounders, with negligible difference between treatment groups (standardized difference $\leq 0.1$ ), ranged between $87 \%$ and $97 \%$ (comprehensive set and domain expert set in BMI outcome, respectively); online supplementary figures $2-5$ display scatter plots of the absolute standardized difference before and after the correction. We found no significant differences between patients on different drug classes when using negative controls of patient height, while finding differences of up to $0.08 \%$ in HbA1c levels before index date between GLP-1 and SU or TZD (see Discussion).

\section{HbA1c outcome}

HbA1c measurements were available for $83 \%$ of the patients from up to 90 days prior to initiation of secondline treatment, and for $95 \%$ of the patients up to 180 days (see online supplementary figure 6 for complete temporal distribution).

The differences in estimated HbAlc levels using the domain expert and comprehensive sets of confounders were lower than $0.03 \%$. All four drug classes were predicted to reduce HbAlc levels below 7\% after 12 months of treatment, with a predicted reduction in HbA1c levels relative to baseline over the entire population of $0.6 \%-0.61 \%$ (SU, domain expert and comprehensive set correction, respectively) to $0.85 \%-0.83 \%$ (GLP-1, domain expert and comprehensive set correction, respectively) (online supplementary table 5, figure 2). Twelve-month HbA1c levels inferred for SU were significantly higher than for TZDs, DPP-4 and GLP-1 by $0.09 \%$ $0.24 \%$ (Wald test, $\mathrm{p}<3 \mathrm{e}^{-5}$; online supplementary table 6). Inferred levels for DPP-4 were significantly higher than TZD after 12 months and higher than GLP-1 after 6 months of treatment, but differences became insignificant after 12 months (online supplementary table 6). Notably, both actual and inferred HbAlc levels were lower than those computed using the mixed-treatment comparison (MTC) of clinical trials of McIntosh et al. ${ }^{78}$

\section{BMI outcome}

BMI measurements were available for $78 \%$ and $83 \%$ of the patients as recent as 90 and 180 days prior to treatment date, respectively (see online supplementary figure 7 for complete time distribution).

The predicted BMI after 12 months was significantly lower for patients on DPP-4 than for patients on SUs or TZDs by $0.47-0.81 \mathrm{~kg} / \mathrm{m}^{2}$ (Wald test, $\mathrm{p}<8 \mathrm{e}^{-4}$; figure 3 , online supplementary figure 7-8), but not significantly lower than the predicted BMI for patients on GLP-1. On average, patients on GLP-1 had higher BMI (by $4.2-4.4 \mathrm{~kg} / \mathrm{m}^{2}$; online supplementary figure 7) before the prescription of second-line treatment compared with patients prescribed one of the other studied drug classes. These GLP-1 patients had lowered their BMI by $0.87 \mathrm{~kg} / \mathrm{m}^{2}$ on average. Our predicted BMI shows significant advantage for GLP-1 over TZD based on the domain expert confounder set, but not statistically significant based on the comprehensive confounder set. It also shows no significant advantage over $\mathrm{SU}$ in a population with lower initial BMI (online supplementary supplementary table 8; see also discussion on BMI and GLP-1).

\section{DISCUSSION}

We presented a causal inference analysis of observational EHR data to compare the effect of adding a second-line treatment for T2DM on HbAlc and BMI, in patients already treated with metformin. Our inferred HbA1c levels for up to 12 months of follow-up suggest that the effect of TZD, DPP-4 and GLP-1 inhibitors is comparable, 


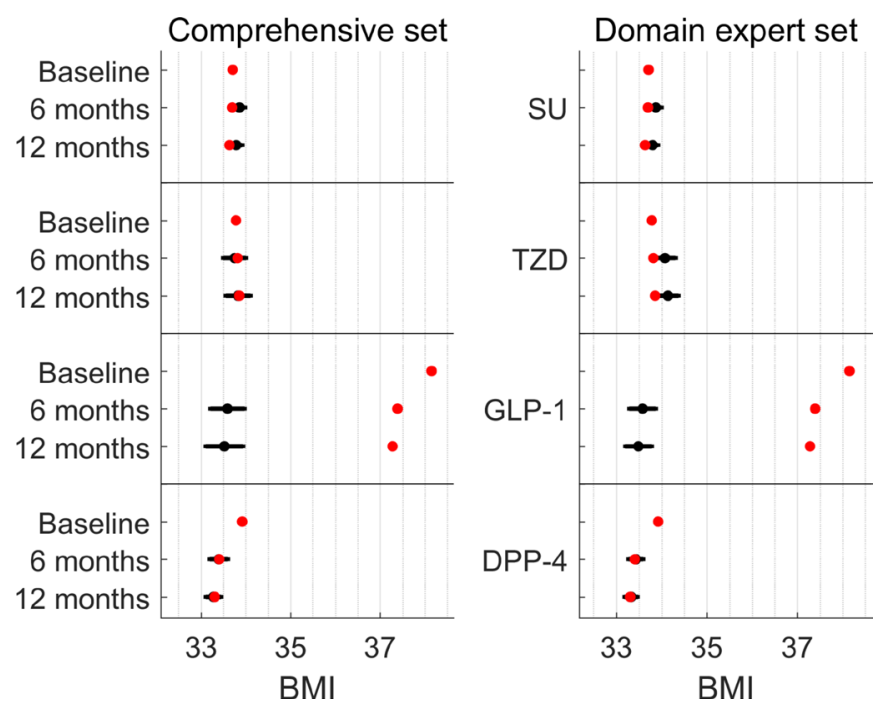

Figure 3 Predicted and observed BMI levels using doubly robust estimation adjusting for either a comprehensive set of confounders (left panel) or a set of confounders provided by a domain expert (right panel). Red dots indicate the actual measurements of patients at baseline (before second-line treatment), after 6 and 12 months. Black dots (with error bars) represent the counterfactual predictions and $95 \% \mathrm{Cls}$, supposing all patients were treated with that drug class. BMI, body mass index; DPP-4, dipeptidyl peptidase 4; GLP-1, glucagon-like peptide-1 receptor agonists; SU, sulfonylurea; TZD, thiazolidinedione.

whereas that of SU is smaller. While TZD and SU have a negligible effect on BMI, DPP-4 and GLP-1 reduce BMI after 12 months of treatment.

The analyzed data contain privately insured employees, Medicare and Medicaid. While the data potentially under-represent children and young adults, as this population is rarely confronted with T2DM, there was no major bias introduced, as can be seen by the comparison of the age distribution in online supplementary figure 1 to the distribution published by the CDC for $2011 .{ }^{31}$

We addressed three challenges associated with causal inference from EHRs, including fragmented data, identification of the true set of confounders, and differences in protocols or adoption rate across institutions. To address fragmented data, we corrected for potential selection bias using patients with incomplete data as censored. In order to reduce the probability of incorrectly specifying confounders or correcting for them, we took the following three measures: For the first measure, we compared confounder sets based on domain expertise with a comprehensive set of confounders based on available clinical and demographic information of the patient to find minor differences in predicted outcomes. We showed the DR estimator improves balancing of confounders for both confounder sets and especially for the domain expert sets, but as noted by Austin ${ }^{29}$ for propensity score models, in many settings it is likely that one can safely include all measured baseline characteristics in the models. For the second measure, we tested whether we could reduce residual confounding by stratifying continuous values, such as age and lab tests, to five categories, as suggested by Groenwold $e t a l^{28}$ Finally, we used DR estimation in order to account for potential misspecification of either the treatment or outcome models. ${ }^{24}$ The DR estimator did not identify difference between drug classes for the negative control of patient height, but did identify small but significant differences in the control of pretreatment HbA1C with regard to GLP-1. This does not affect the overall conclusions of the paper, but suggests that with regard to GLP-1, pretreatment HbA1C cannot be fully explained by other confounders.

Explorys database is an amalgamation of patient data from multiple clinics. While the data have undergone standardization and normalization procedures to account for the differences between healthcare facilities, our estimated effect sizes might deviate from the true effects in individual healthcare facilities. Additionally, we could not account for potential environmental confounders that are not available in EHR data, such as lifestyle changes.

We consider HbAlc and BMI as good proxies for future patient risk, ${ }^{32}$ but there are other considerations in selecting a second-line drug beyond its effect on these measures, such as risks of adverse reactions and of diabetes-related complications. While we did not directly address adverse reactions, patients who were switched drug classes may indirectly point to such effects. These outcomes should be studied in subsequent work, potentially observing patients for longer follow-up periods to gain stronger statistical power. Other extensions should focus on differences between individual drugs from the same class, which could have different outcomes (such as different drugs from the SU class $^{33}$ ).

Patients prescribed SU were less likely to be added a third drug or switched to another drug than patients on the other drugs studied ( $16 \%$ of the patient on SU relative to $>24 \%$ of the patients on the other three drug classes, $\mathrm{p}<\mathrm{e}^{-150}$ ). This is despite the effect of SU on HbAlc being somewhat smaller. Possible explanations for this observation may include the low cost of SU, the availability of a metformin-SU pill ${ }^{34}$ and the option of once-a-day dosing. Additionally, SU and TZD patients had significantly lower availability of BMI measurements during the follow-up period. Possible explanations for this are that when GLP-1 or DPP-4 treatments are prescribed, either the physician or the patient is more likely to have been concerned with the BMI, thus measures it more frequently; or that costs of SU and TZD tend to be lower and would be more frequently prescribed to patients with a lower socioeconomic state, which tend to be less well followed up on.

There may have been some unmodeled confounding present in the relationship between GLP-1 and HbA1C, considering that a small but significant association arose with the negative control of pretreatment HbA1C. We note that GLP-1 agonists are prescribed significantly more to women. Difference in response to GLP-1 between men and women was reported in $2005,{ }^{35}$ and a study from 2013 found that the effect of one such GLP-1 agonist, exenatide, was larger in women. ${ }^{36}$ All patients in 
our study were treated with GLP-1 after 2005 and $38 \%$ of them treated during or after 2013, suggesting physicians may have considered this evidence when prescribing GLP-1. Also, patients on GLP-1 are typically younger than patients on other drug classes, in line with an observation made by others. ${ }^{37}$ Finally, patients with higher BMI tend to be prescribed GLP-1, and this is likely due to its known positive effect on weight. ${ }^{38} 39$ In our analysis, though, DPP-4 inhibitors are estimated to lead to BMI reduction comparable to GLP-1 agonists.

TZD is the only class predicted to maintain HbAlc at a stable level in 6 and 12 months, whereas HbAlc levels are predicted to increase over time in the other studied classes. A gradual weaning of the effect of SU on HbAlc levels had been previously described. ${ }^{13}$

The estimates of $\mathrm{HbAl}^{8}$ reported in the meta-analysis (MTC) we used for reference were higher than our EHR-based inference. We note that we predicted HbAlc in exact periods, while the MTC method combined heterogeneous time point measurements across the different clinical trials, some listed as having up to 5 years of follow-up. This may suggest that the meta-analysis captured later stages in the progression of T2DM, characterized by higher HbAlc levels. ${ }^{40}$

As demonstrated by our analysis, as well as by others, ${ }^{41}$ EHR data can support causal inference and allow replication of clinical trial results. The advantages of this approach in terms of the labor and costs required to expand evidence-based medicine are clear. As the availability of EHR data increases and the many theoretical and technical challenges associated with detecting and correcting for confounders are addressed, we expect causal inference based on observational data to become more widely used.

Acknowledgements We would like to thank Omer Weissbrod for helpful inputs and suggestions, Michal Ozery-Flato for help with feature engineering tasks, and the anonymous reviewers for the exceptionally constructive comments. We are also grateful to the Explorys team, and in particular to Euricka S Thomas and Gabriel Olinger, for the continued support and advice.

Contributors $A G$ and $C Y$ designed and performed the experiments. AC compiled domain expert confounders. AG, CY, AC and YG wrote the manuscript. AG and $C Y$ are guarantors of the paper.

Competing interests None declared.

Provenance and peer review Not commissioned; externally peer reviewed. Data sharing statement № additional data are available.

Open Access This is an Open Access article distributed in accordance with the Creative Commons Attribution Non Commercial (CC BY-NC 4.0) license, which permits others to distribute, remix, adapt, build upon this work non-commercially, and license their derivative works on different terms, provided the original work is properly cited and the use is non-commercial. See: http://creativecommons.org/ licenses/by-nc/4.0/

(c) Article author(s) (or their employer(s) unless otherwise stated in the text of the article) 2017. All rights reserved. No commercial use is permitted unless otherwise expressly granted.

\section{REFERENCES}

1. Centers for Disease Control and Prevention. National diabetes statistics report: estimates of diabetes and its burden in the
United States, 2014 [Internet]. http://www.cdc.gov/diabetes/pubs/ statsreport14/national-diabetes-report-web.pdf (accessed $16 \mathrm{Nov}$ 2016).

2. WHO. Global report on diabetes, 2016 [Internet]. http://apps.who.int/ iris/bitstream/10665/204871/1/9789241565257_eng.pdf (accessed 16 Nov 2016).

3. American Diabetes Association. Standards of Medical Care in Diabetes 2016 [Internet]. http://care.diabetesjournals.org/content/ suppl/2015/12/21/39.Supplement_1.DC2/2016-Standards-of-Care. pdf (accessed 16 Nov 2016).

4. Rojas LB, Gomes MB. Metformin: an old but still the best treatment for type 2 diabetes. Diabetol Metab Syndr 2013;5:6.

5. Berkowitz SA, Krumme AA, Avorn J, et al. Initial choice of oral glucose-lowering medication for diabetes mellitus: a patientcentered comparative effectiveness study. JAMA Intern Med 2014;174:1955-62.

6. Kamenov Z. Effectiveness and tolerability of second-line therapy with vildagliptin versus other oral agents in type 2 diabetes (EDGE): post hoc sub-analysis of Bulgarian data. Diabetes Ther 2014:5:483-98

7. Phung OJ, Scholle JM, Talwar M, et al. Effect of noninsulin antidiabetic drugs added to metformin therapy on glycemic control, weight gain, and hypoglycemia in type 2 diabetes. JAMA 2010;303:1410-8.

8. Mclntosh B, Cameron C, Singh SR, et al. Second-line therapy in patients with type 2 diabetes inadequately controlled with metformin monotherapy: a systematic review and mixed-treatment comparison meta-analysis. Open Med 2011;5:e35-48.

9. Monami M, Lamanna C, Marchionni N, et al. Comparison of different drugs as add-on treatments to metformin in type 2 diabetes: a metaanalysis. Diabetes Res Clin Pract 2008;79:196-203.

10. Cefalu WT, Buse JB, Del Prato S, et al. Beyond metformin: safety considerations in the decision-making process for selecting a second medication for type 2 diabetes management. Diabetes Care 2014;37:2647-59.

11. Downing NS, Aminawung JA, Shah ND, et al. Clinical trial evidence supporting FDA approval of novel therapeutic agents, 2005-2012. JAMA 2014;311:368-77.

12. Greco T, Zangrillo A, Biondi-Zoccai G, et al. Meta-analysis: pitfalls and hints. Heart Lung Vessel 2013;5:219-25.

13. Cook MN, Girman CJ, Stein PP, et al. Glycemic control continues to deteriorate after sulfonylureas are added to metformin among patients with type 2 diabetes. Diabetes Care 2005;28:995-1000.

14. Stuart EA, DuGoff E, Abrams M, et al. Estimating causal effects in observational studies using electronic health data: challenges and (some) solutions. EGEMS 2013;1:4.

15. Valdes I, Kibbe DC, Tolleson G, et al. Barriers to proliferation of electronic medical records. Inform Prim Care 2004;12:3-9.

16. Brookhart MA, Stürmer T, Glynn RJ, et al. Confounding control in healthcare database research: challenges and potential approaches. Med Care 2010;48:S114-20.

17. Nair S, Hsu D, Celi LA. Challenges and opportunities in secondary analyses of electronic health record data. Critical Data MIT, ed. Secondary analysis of electronic health records [Internet]. Cham: Springer International Publishing, 2016:17-26.

18. Pacheco J, Thompson W. Type 2 diabetes mellitus PheKB [Internet]: Northwestern University, 2012. https://phekb.org/phenotype/type-2diabetes-mellitus (accessed 27 Nov 2016).

19. Ozery-Flato M, Yanover C, Gottlieb A, et al. Fast and efficient feature engineering for multi-cohort analysis of EHR data. Stud Health Technol Inform 2017;235:181-5.

20. Charlson ME, Pompei P, Ales KL, et al. A new method of classifying prognostic comorbidity in longitudinal studies: development and validation. J Chronic Dis 1987;40:373-83.

21. Elixhauser A, Steiner C, Harris DR, et al. Comorbidity measures for use with administrative data. Med Care 1998;36:8-27.

22. Marginal Structural Models and Causal Inference in Epidemiology. Robins, Hernan and Brumback 2000 marginal structural models and causal.pdf [Internet]. http://www.epidemiology.ch/history/ PDF\%20bg/Robins, \%20Hernan\%20and\%20Brumback\%202000\% 20 marginal\%20structural\%20models\%20and\%20causal.pdf (accessed 27 Nov 2016)

23. Robins J, Sued M, Lei-Gomez Q, et al. Comment: performance of double-robust estimators when "Inverse Probability" weights are highly variable. Statistical Science 2007;22:544-59.

24. Bang $\mathrm{H}$, Robins JM. Doubly robust estimation in missing data and causal inference models. Biometrics 2005;61:962-73.

25. Gerhard T, Delaney JA, Cooper-Dehoff RM, et al. Comparing marginal structural models to standard methods for estimating treatment effects of antihypertensive combination therapy. BMC Med Res Methodol 2012;12:119. 
26. Cole SR, Hernán MA. Constructing inverse probability weights for marginal structural models. Am J Epidemiol 2008;168:656-64.

27. Frölich M. Finite-sample properties of propensity-score matching and weighting estimators. Rev Econ Stat 2004;86:77-90.

28. Groenwold $\mathrm{RH}$, Klungel OH, Altman DG, et al. Adjustment for continuous confounders: an example of how to prevent residual confounding. CMAJ 2013;185:401-6.

29. Austin PC. An introduction to propensity score methods for reducing the effects of confounding in observational studies. Multivariate Behav Res 2011;46:399-424.

30. Lipsitch M, Tchetgen Tchetgen E, Cohen T. Negative controls: a tool for detecting confounding and bias in observational studies. Epidemiology 2010;21:383-8.

31. Centers for Disease Control and Prevention. Distribution of age at diagnosis of diabetes among adult incident cases aged 18-79 years, United States, 2011. Dep Health Hum Serv 2009:1-2.

32. American Diabeties Association. HbA1c as a predictor of diabetes and as an outcome in the diabetes prevention program: a randomized clinical trial | diabetes care [Internet]. http://care. diabetesjournals.org/content/38/1/51 (accessed 27 Nov 2016).

33. Lyford TPJ 3 N 2014By J. Mortality risk should be considered with choice of sulfonylurea drug [Internet]. Pharmaceutical Journal http:// www.pharmaceutical-journal.com/news-and-analysis/mortality-riskshould-be-considered-with-choice-of-sulfonylurea-drug/20066979. article (accessed 27 Nov 2016).
34. Sola D, Rossi L, Schianca GP, et al. Sulfonylureas and their use in clinical practice. Arch Med Sci 2015;11:840-8.

35. Adam TC, Westerterp-Plantenga MS. Nutrient-stimulated GLP1 release in normal-weight men and women. Horm Metab Res 2005;37:111-7.

36. Anichini R, Cosimi S, Di Carlo A, et al. Gender difference in response predictors after 1-year exenatide therapy twice daily in type 2 diabetic patients: a real world experience. Diabetes Metab Syndr Obes 2013;6:123-9.

37. Conget I, Mauricio D, Ortega R, et al. Characteristics of patients with type 2 diabetes mellitus newly treated with GLP-1 receptor agonists (CHADIG Study): a cross-sectional multicentre study in Spain. BMJ Open 2016;6:e010197.

38. Marre $M$, Shaw J, Brändle $M$, et al. Liraglutide, a once-daily human GLP-1 analogue, added to a sulphonylurea over 26 weeks produces greater improvements in glycaemic and weight control compared with adding rosiglitazone or placebo in subjects with type 2 diabetes (LEAD-1 SU). Diabet Med 2009;26:268-78.

39. Astrup A, Carraro R, Finer N, et al. Safety, tolerability and sustained weight loss over 2 years with the once-daily human GLP-1 analog, liraglutide. Int J Obes 2012;36:843-54.

40. Fonseca VA. Defining and characterizing the progression of type 2 diabetes. Diabetes Care 2009;32(Suppl 2):S151-S156.

41. Kleinberg S, Hripcsak G. A review of causal inference for biomedical informatics. J Biomed Inform 2011;44:1102-12. 